\title{
NOISE ESTIMATION OF HYPERSPECTRAL REMOTE SENSING IMAGE BASED ON MULTIPLE LINEAR REGRESSION AND WAVELET TRANSFORM
}

Estimativas dos ruidos nas imagens hiperespectrais de Sensoriamento Remoto baseadas na regressão linear múltipla e transformada "wavelet"

\author{
DONG XU \\ LEI SUN \\ JIANSHU LUO
}

College of Science, National University of Defence Technology Changsha, Hunan, P. R. China 410073

E-mail: xu_don@163.com; bangbangbing1999@163.com; ljsh3115@sina.com

\begin{abstract}
Noise estimation of hyperspectral remote sensing image is important for its post-processing and application. In this paper, not only the spectral correlation removing is considered, but the spatial correlation removing by wavelet transform is considered as well. Therefore, a new method based on multiple linear regression (MLR) and wavelet transform is proposed to estimate the noise of hyperspectral remote sensing image. Numerical simulation of AVIRIS data is carried out and the real data Hyperion is also used to validate the proposed algorithm. Experimental results show that the method is more adaptive and accurate than the general MLR and the other classified methods.

Keywords: Hyperspectral Remote Sensing Image; Wavelet Transform; MLR, Signal-to-Noise Ratio (SNR).

\section{RESUMO}

Estimativa do ruído de imagens hiperespectrais de sensoriamento remote é importante para o pós-processamento e aplicações. Nesta pesquisa, não somente a remoção da correlação espectral é considerada, como também a remoção da correlação espacial pela transformada wavelet . Portanto, um novo método baseado na regressão linear múltipla (MLR) com a transformada wavelet é proposto para


estimar o ruído da imagem hiperespectrais de sensoriamento remoto. Uma simulação numérica de dados AVIRIS é executada, e dados reais Hyperion são utilizados para validar o algoritmo proposto. Resultados experimentais mostram que o método é mais adaptável e preciso do que o MLR geral e outros métodos classificados.

Palavras-Chave: Imagem de Sensoriamento Remoto Hiperespectral; Transformada Wavelet; MLR; Sinal de ruído médio.

\section{INTRODUCTION}

Hyperspectral remote sensing images can be viewed as $n$-dimensional data consisting of one-dimensional spectral information and two-dimensional spatial information (RICHARDS and JIA, 1999). With the fast development of hyperspectral remote sensing technology, hyperspectral remote sensing images can describe the characteristics of Earth objects more comprehensive and explicitly, therefore, they are widely applied in many fields including agriculture, forestry, geological surveys, environmental monitoring, military reconnaissance etc. When sunlight travels from the sun to the Earth's surface, through the atmosphere and then to the sensor, the atmosphere often scatters some light, the electromagnetic radiation propagation path of hyperspectral imaging will be subjected by the effect of many complex factors, thus a lot of noise is introduced, which brings a negative impact on the image analysis. The noise type and parameters are quite important for its postprocessing and application, so it is very necessary to study the noise estimation of hyperspectral remote sensing image.

Hyperspectral remote sensing images are three-dimensional images. Comparing with normal three-dimensional data cube of fixed variance of additive noise, the noise level of hyperspectral image may vary dramatically from band to band. The noise standard deviation in each band of hyperspectral remote sensing image is not constant, in particular, there exist some bands at which the atmosphere absorbs so much light that the signal received from the surface is unreliable (RICHARDS and JIA,1999). The noise model mentioned by many references (BRUONO AIAZZI, et al, 2006; USS, et al, 2011; ACTIO, et al, 2011; MEOLA, et al, 2011) is composed of both the signal-dependent noise and the signal-independent noise, but in many references (GAO, 1993; ROGER and ARNOLD, 1996; GAO, et al, 2008; JULIO, 2008; CHEN and QIAN, 2011) the signal-independent noise is viewed as the dominant component. This paper is focus on the signal-independent noise.

In this paper, the noise term is modeled as additive and spatially stationary in each band, but the variance of the noise varies from band to band. That is to say, the level of the noise is dependent on the average amplitude of each band, but spatially stationary in each band. Recently, several noise estimation methods based on this noise model are proposed. The whole image is artificially divided into small blocks to evaluate the local deviation, and then the deviation of the entire image is the 
mean of the local deviations with the same weight (GAO, 1993). Between-band (spectral) and within-band (spatial) correlations (SSDC) method is automatic and does not require the intervention of a human operator and the noise is estimated from the residuals of the multiple linear regression (MLR) (ROGER and ARNOLD, 1996). Gao, et al (2008) proposed that a hyperspectral image is classified into blocks by an algorithm based on the internal regularity of the Earth object and the strong spectral correlation of hyperspectral remote sensing image (GENG, et al 2004). Recursive Core (RC) method proposed by Julio (2008) is used to prevent the side effects of the defective segmentation process of the method proposed by Gao et al (2008). The RC method is a suitable fix for the region growing stage. MLR is performed in each class. The best noise estimate of the entire image is the mean of the standard deviation of all the classes. The general MLR method based on the different number of bands has been introduced (BIOUCAS-DIAS, et al, 2008; ACITO, et al, 2011). This method is called "general MLR" in the remainder of this paper.

The above algorithms are based on the classification of hyperspectral data using the characteristic of Earth object. The classification can be viewed as an approach to remove the spatial correlation of hyperspectral data, and MLR is applied to each class. The residual image obtained by MLR is considered to be the noise. All of these methods firstly remove the spatial correlation by classification, and then remove the spectral correlation by MLR. Therefore, the accuracy of the estimation by the classification algorithms depends on the Earth objects. In recent years, wavelet proposed by Mallat (1989) has been widely used for image processing. Wavelet has a good time-frequency-localization property. As a result of multi-resolution approach, the signal power is concentrated in the low frequency subband by wavelet transform, and the high frequency subband describes nonstationary characteristics of the signal well. Thus wavelet transform can accurately capture the significant information about an object of interest using a sparse description, and remove the spatial correlation of the image. Therefore, this paper presents a noise estimation method of hyperspectral remote sensing image, which is based on MLR and wavelet transform. The method removes the spatial correlation of the residual image obtained by MLR via wavelet transform. At last the standard deviation is estimated from the median absolute value of the wavelet coefficients of the noisy signals in the high-frequency subbands. The experiments are tested on both simulated and real data. The simulated experiment is tested on AVIRIS data added with Gaussian white noise and real data experiment is tested on Hyperion data. Results show that the method can estimate the standard deviation of the noise in each band accurately.

The structure of this paper is as follows, section 2 describes the noise estimation based on MLR model and wavelet transform, section 3 gives the experiment of results by comparison with both general MLR and classification method, section 4 draws conclusion. 
2. NOISE ESTIMAION OF HYPERSPECTRAL REMOTE SENSING IMAGE

\subsection{MLR Model for Hyperspectral Remote Sensing Image}

MLR is widely used to remove the spectral correlation from hyperspectral image. In order to remove the spectral correlation of hyperspectral remote sensing image, we use $L$ adjacent bands to predict the pixels in band $k$ by MLR. We assume that each band has $M \times N$ pixels. Let $\mathbf{X}$ denote a $P \times B$ matrix of the $B$ spectral observed vectors of size $P(P=M \times N)$. In this paper, the $P \times 1$ vector $\mathbf{X}_{k}$ is the $k$-th column vector of the matrix $\mathbf{X} . \hat{\mathbf{X}}_{k}$ is vector predicted for the signal $\mathbf{X}_{k}$ of band $k$ pixel. The $L$ adjacent bands are utilized to perform MLR. That is

$$
\begin{gathered}
\hat{\mathbf{X}}_{k}=\mathbf{X}_{\lambda k} \boldsymbol{\beta}_{k} \\
\hat{\boldsymbol{\xi}}_{k}=\mathbf{X}_{k}-\hat{\mathbf{X}}_{k}(k=1,2, \ldots, B)
\end{gathered}
$$

where the $P \times L$ matrix $\mathbf{X}_{\lambda k}$ is consisted of the $L$ column vectors of $\mathbf{X}$ (not including the $k$-th column vector), $\mathbf{X}_{\lambda k}=\left[\mathbf{X}_{1}, \cdots, \mathbf{X}_{k-1}, \mathbf{X}_{k+1}, \cdots, \mathbf{X}_{L+1}\right] . \quad \boldsymbol{\beta}_{k}$ is the regression vector of size $L \times 1$, and $\hat{\xi}_{k}$ is the $k$-th column vector of the residual image, here, $\hat{\xi}=\left[\hat{\xi}_{1}, \hat{\xi}_{2}, \ldots, \hat{\xi}_{B}\right]$. For $k=1,2, \ldots, B$, the least squares estimator of the regression vector $\boldsymbol{\beta}_{k}$ is given by

$$
\hat{\boldsymbol{\beta}}_{k}=\left(\mathbf{X}_{\lambda k}^{\mathrm{T}} \mathbf{X}_{\lambda k}\right)^{-1} \mathbf{X}_{\lambda k}^{\mathrm{T}} \mathbf{X}_{k}
$$

The noise is extracted from the residual image

$$
\hat{\xi}_{k}=\mathbf{X}_{k}-\mathbf{X}_{\lambda k} \hat{\boldsymbol{\beta}}_{k}
$$

\subsection{Noise Estimation by Wavelet Transform}

The spectral correlation of hyperspectral remote sensing image by MLR can be removed effectively, but the spatial correlation still remains in the residual image. Therefore it is necessary to remove the spatial correlation of the residual image and then the level of the noise can be better estimated. In this paper we use wavelet transform to remove the spatial correlation, and then estimate the standard deviation of image noise. Wavelet transforms the image into a new presentation domain by multi-scale transform, and decouples the higher-order statistical characteristic of natural images, so the signal power is concentrated in the lowfrequency subbands of the wavelet coefficients, on the contrary the high-frequency subbands of the wavelet coefficients describe the signals which change sharply or are discontinuous. Since the wavelet transform is orthogonal, Gaussian white noise is scattered throughout the wavelet coefficients domain after the orthogonal transformation, and obeys a Gaussian distribution in the new representation domain. Due to the statistical properties of the images in wavelet domain, we use the median 
absolute deviation (MAD) to estimate the level of the noise standard deviation (Donoho and Johnstone, 1994). MAD is as follows:

The signal $x$ can be represented as $x=\sum_{\lambda \in \Lambda^{L}} d_{\lambda} \psi_{\lambda}$ by wavelet transform, where subscript $\lambda=(i, j, l)$ stands for the coefficient of position $(i, j)$ in the $l$-th scale. MAD estimates the level of the noise by taking the median of the modulus of the fine scale wavelet coefficients. The noise standard deviation $\tilde{\sigma}$ is estimated as

$$
\tilde{\sigma}=\frac{1}{0.6745} \underset{\lambda=(i, j, l) \in\{(i, j, l), l=2,3, \ldots, L\}}{\text { Median }_{\lambda}}\left(\left|d_{\lambda}\right|\right)
$$

where Median $\left(\left|d_{\lambda}\right|\right)$ means to take the median of $\left|d_{\lambda}\right|$, and the term 0.6745 results from the reciprocal of the normal inverse cumulative distribution function $\Phi^{-1}(p)$ evaluated at probability $p=0.75$.

Estimating the variance of the noise by assuming that "most" of the empirical wavelet coefficients at each resolution level are noise, and hence that MAD reflects the level of the typical noise (DONOHO, 1993).

\subsection{Noise Estimation Based on Multiple Linear Regression Model and Wavelet \\ Transform (MLRWT)}

As there is strong linear correlation between the bands of hyperspectral remote sensing image, the spectral correlation can be removed successfully by MLR. The residual image obtained by MLR maintains some features of hyperspectral remote sensing image. Wavelet transform is performed on the residual image obtained by MLR, so that the spatial correlation of images can be removed, that is, the noise and the features can be separated. At last MAD is employed to estimate the noise standard deviation.

The algorithm processes used in this paper as follows (Figure 1):

1. Input the hyperspectral remote sensing data $\mathbf{X}$, where the size of the data is $M \times N \times B$ (width $\times$ height $\times$ band).

2. For $k=1,2, \ldots, B$, band $k$ residual image $\hat{\xi}_{k}$ is predicted by MLR

3. For $k=1,2, \cdots B$, perform wavelet decomposition (WD) for residual image $\hat{\xi}_{k}$ to obtain fine scale wavelet coefficients $\mathbf{d}_{\lambda}^{k}$, and use the equation (6) to estimate the noise standard deviation of each band

$$
\tilde{\sigma}_{k}=\frac{1}{0.6745} \operatorname{Median}\left(\left|\mathbf{d}_{\lambda}^{k}\right|\right)
$$

Figure 1 - Block diagram of the proposed method in this paper.

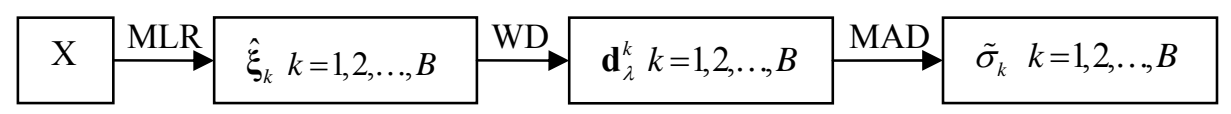




\section{EXPERIMENTAL RESULTS}

\subsection{Simulated experiment}

The simulated experiment of the noise estimation is carried out on AVIRIS image, Jasper Ridge (Scene Request ID $=$ f970403t01p02_r03, Site Latitude $=+37.5$, Site Longitude $=-122.3$, Date: 03/04/97) provided by JPL, NASA. The size of datacube we extracted from the Jasper Ridge for testing is $256 \times 256 \times 224$ (width $\times$ height $\times$ band). Figure 2(a) shows the image in band 40. An important parameter to measure the image quality is signal-to-noise ratio (SNR). Here, the SNR is defined as follows

$$
S N R=10 \log _{10}\left(\frac{P_{X}}{P_{N}}\right)
$$

Figure 2 - Band 40 of (a) Jasper Ridge and (b) Hyperion data.

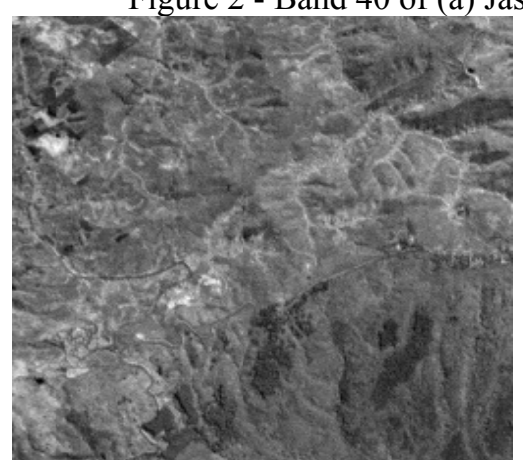

(a)

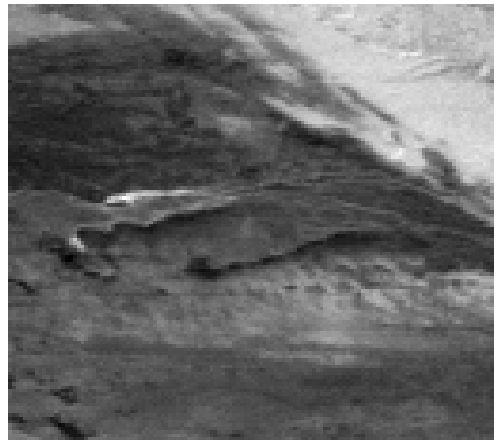

(b)

where $P_{X}$ is the power of the pure signals $\mathbf{X}_{i, j}^{k}$, and $P_{N}$ is the noise power in the noisy signals $\tilde{\mathbf{X}}_{i, j}^{k}$, where $(i, j, k)$ stands for the position $(i, j)$ in the $k$-th band.

$$
S N R=10 \log _{10}\left(\frac{\sum_{i=1, j=1, k=1}^{M, N, B}\left|\mathbf{X}_{i, j}^{k}\right|^{2}}{\sum_{i=1, j=1, k=1}^{M, N, B}\left|\tilde{\mathbf{X}}_{i, j}^{k}-\mathbf{X}_{i, j}^{k}\right|^{2}}\right)
$$

For the AVIRIS image a $28 m \times 28 m$ ground sample distance (GSD) datacube is derived by spatially averaging the $4 m \times 4 m$ GSD datacube elevating the nominal SNR to $38.45 \mathrm{~dB}$. Having such high SNR, this datacube is viewed as a pure datacube (Othman and Qian, 2006). The noise model is given according to the reference. The images are corrupted by Gaussian white noise. It is different from the simple stationary additive noise model that is simulated by adding noise with a fixed standard deviation to the datacube, the noise variance is proportional to the average 
signal amplitude of each band, but the noise in each band is still additive noise. The SNR of the simulated noisy data is $27.78 \mathrm{~dB}$, which is chosen by comprehensive requirement of the users and the machine design parameters. The difference between the pure image and the noise image is so subtle that it is difficult to distinguish by the human eyes, but has a big impact on the final remote sensing products and application. So it is important to estimate such subtle noise accurately.

The estimated noise standard deviation is denoted by $\tilde{\sigma}_{k}$ and the standard deviation of the simulated noise is denoted by $\sigma_{k}, k=1,2, \ldots, 224$. In order to compare with the other methods, the maximum, the minimum and the average error used in this paper are defined as $\max _{k=1, \ldots, 224}\left|\tilde{\sigma}_{k}-\sigma_{k}\right|, \min _{k=1, \ldots, 224}\left|\tilde{\sigma}_{k}-\sigma_{k}\right|$ and $\frac{1}{224}\left(\sum_{k=1}^{224}\left|\tilde{\sigma}_{k}-\sigma_{k}\right|\right)$, respectively.

To choose the proper parameters for wavelet transform, different levels of decomposition and types of wavelets are tested on the simulated data Jasper Ridge. Six levels of wavelet decomposition achieve good performance in this experiment. Two types of wavelet families are implemented, $\mathrm{db} N$ and Coif $N$ wavelets, where $N$ is the order of the wavelet function. Table 1 lists the maximum error, minimum error and average error of estimation using different wavelets with six levels of decomposition and different number of bands. By comprehensive consideration of the average error, the maximum error and the minimum error, wavelet $\mathrm{db} 5$ with six levels of decomposition is chosen in the following experiment.

Table 1 - The errors of estimation using different wavelet with six levels of decomposition and different number of bands.

\begin{tabular}{c|c|c|c|c|c|c}
\hline $\begin{array}{c}\text { The } \\
\text { type of } \\
\text { wavelet }\end{array}$ & $\begin{array}{c}\text { Maximum } \\
\text { error }\end{array}$ & $\begin{array}{c}\text { Minimum } \\
\text { error }\end{array}$ & $\begin{array}{c}\text { Average } \\
\text { error }\end{array}$ & $\begin{array}{c}\text { Maximum } \\
\text { error }\end{array}$ & $\begin{array}{c}\text { Minimum } \\
\text { error }\end{array}$ & $\begin{array}{c}\text { Average } \\
\text { error }\end{array}$ \\
\hline db1 & 32.1118 & 0.1948 & 2.3627 & 31.5763 & 0.2671 & 2.5812 \\
db2 & 31.4440 & 0.0298 & 2.1947 & 31.1728 & 0.1834 & 2.4070 \\
db3 & 31.0402 & 0.0225 & 1.8903 & 30.7405 & 0.0148 & 2.0836 \\
db4 & 30.0803 & 0.0055 & 1.6316 & 29.6847 & 0.0759 & 1.7626 \\
db5 & 29.4718 & 0.0018 & $\mathbf{1 . 5 6 9 9}$ & 29.0474 & 0.0049 & $\mathbf{1 . 6 2 6 0}$ \\
db6 & 30.3913 & 0.0110 & 1.6670 & 29.7663 & 0.0473 & 1.8195 \\
db7 & 30.7391 & 0.0098 & 1.7821 & 30.4062 & 0.0395 & 1.9753 \\
db8 & 30.6063 & 0.0011 & 1.7353 & 30.4414 & 0.0200 & 1.9159 \\
db9 & 31.6551 & 0.1083 & 1.9573 & 31.2266 & 0.2552 & 2.1612 \\
db10 & 31.4444 & 0.1141 & 2.0741 & 31.0684 & 0.2700 & 2.2742 \\
coif2 & 31.0043 & 0.0745 & 1.9346 & 30.5084 & 0.1124 & 2.1372 \\
coif3 & 31.0736 & 0.0019 & 1.8989 & 30.6212 & 0.0703 & 2.1168 \\
coif4 & 30.7886 & 0.0061 & 1.8928 & 30.3909 & 0.1603 & 2.1061 \\
\hline
\end{tabular}


Table 2 - Comparison of the errors of noise estimation by MLRWT, MLR and RC.

\begin{tabular}{c|c|c|c}
\hline & Maximum error & Minimum error & Average error \\
\hline MLRWT & 29.4718 & 0.0018 & 1.5699 \\
\hline RC & 27.7704 & 0.1453 & 2.5953 \\
\hline MLR223 & 32.2104 & 0.4017 & 2.6188 \\
\hline MLR50 & 32.1117 & 0.5149 & 2.9344 \\
\hline
\end{tabular}

In the simulated experiment, the comparison between MLRWT and MLR using 223 bands (BIOUCAS-DIAS, et al, 2008) and 50 bands $(0.2 \cdot M, M$ is the number of samples) (ACITO, et al, 2011) respectively is performed. Figure 3 shows the difference between the standard deviation of the two methods and the standard deviation of the simulated noise. The result shows that it is important to reduce the spatial correlation by wavelet after the spectral correlation removing step to improve the accuracy of noise estimation. Meanwhile, it shows that MLR223WT obtains a better result. So MLR223WT is referred to as MLRWT, hereinafter.

In order to illustrate the superiority of the proposed algorithm in our paper, our method is also compared with the algorithm RC proposed by Julio (2008). Table 2 lists the estimated standard deviation of the noise in Jasper Ridge by the general MLR using different numbers of bands, RC and MLRWT, respectively.

In the RC method based on classification, the spectral similarity threshold plays an important role in the performance. The spectral angle distance is taken as the spectral similarity threshold in this paper for it is one of the most important parameters to measure the similarity between the two spectrums (KESHAVA and MUSTARD 2002). By previous tuning of the spectral similarity threshold, the RC method obtains an optimal result by the threshold 0.0675 for the Jasper Ridge and the image scene is divided into 61 classes.

On the contrary, MLRWT does not need such tuning, and demonstrates better adaptability and a substantial improvement in the estimation accuracy. By comparing with the general MLR using different numbers of bands, the result shows that wavelet transform reduces the spatial correlation successfully and provide more accurate estimation.

From Table 2, compared with the general MLR, and the RC method, the estimation accuracy of the Jasper Ridge is obviously improved by MLRWT in the terms of the average error. From Figure 4(a), it is difficult to see the comparative result, but it can be observed from Figure 4(b) that our method provides better noise estimation results. It also indicates that the combination of MLR and WT is avaliable. 
Figure 3 - The difference between the standard deviation of simulated noise and the estimated standard deviation by MLRWT and MLR. MLR223 and MLR50 mean that the MLR utilizes 223 bands and 50 bands (not including itself) respectively for regression.

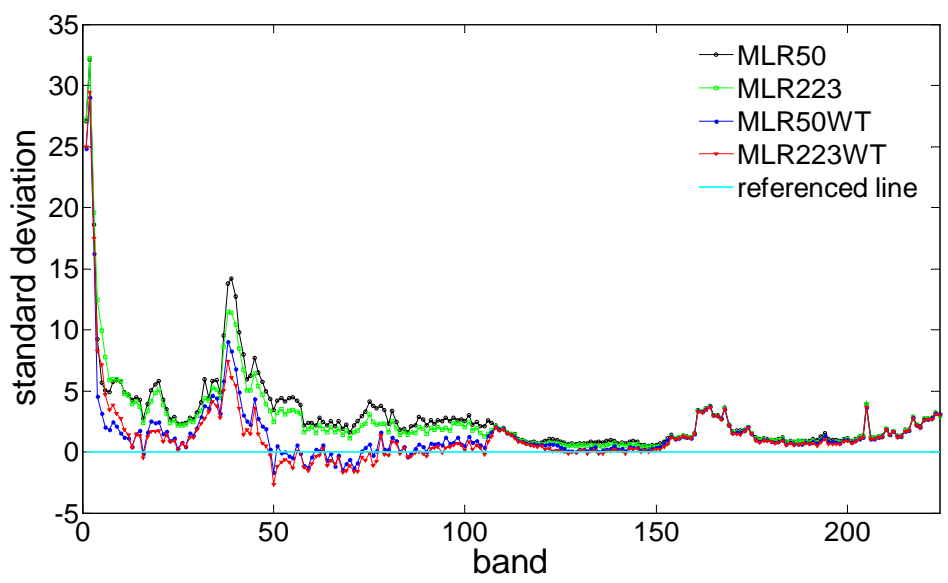

Figure 4 - The estimated noise standard deviation of MLRWT, the general MLR and $\mathrm{RC}$ for the Jasper Ridge, (b) the difference between the standard deviation of simulated noise and the estimated standard deviation by MLRWT and RC.

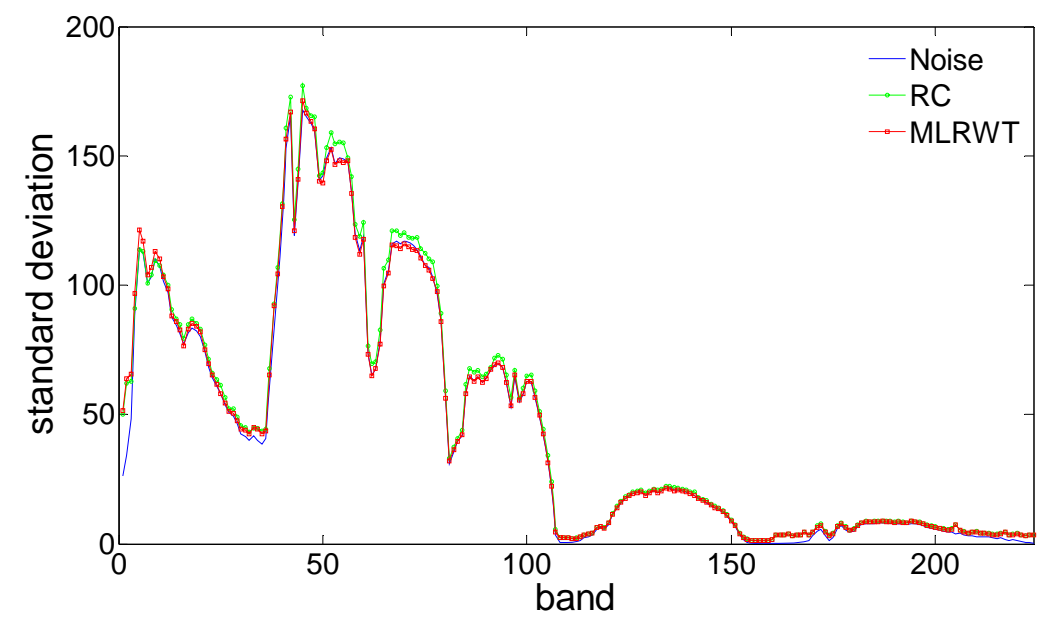

(a) 


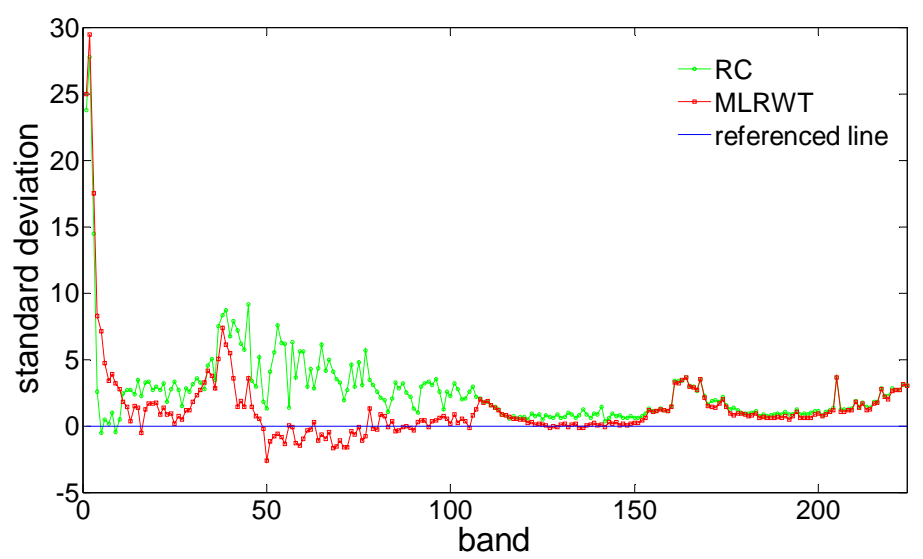

(b)

\subsection{Real data experiment}

The real data experiment of the noise estimation is carried out on Hyperion data (Scene Request ID=EO11280292010303110KF, Site Latitude $=+44.1$, Site Longitude $=+109.6$, Date: 03/03/10). The data is from the web: http://datamirror.csdb.cn/. Hyperion covers the $0.4-2.5 \mu \mathrm{m}$ range with 242 spectral bands at approximately $10-\mathrm{nm}$ spectral resolution and $30-\mathrm{m}$ spatial resolution (KRUSE, et al, 2003). As there are some bands in which all the pixel values are zero, these bands are discarded in our experiment. Therefore, the size of datacube we extract from the Hyperion data for testing is $128 \times 128 \times 198$ (width $\times$ height $\times$ band). Figure 2(b) shows band 40 of this data.

Figure 5 - The estimated noise standard deviation of MLRWT, the general MLR and $\mathrm{RC}$ for the Hyperion data. The gaps refer to the bands discarded in the test.

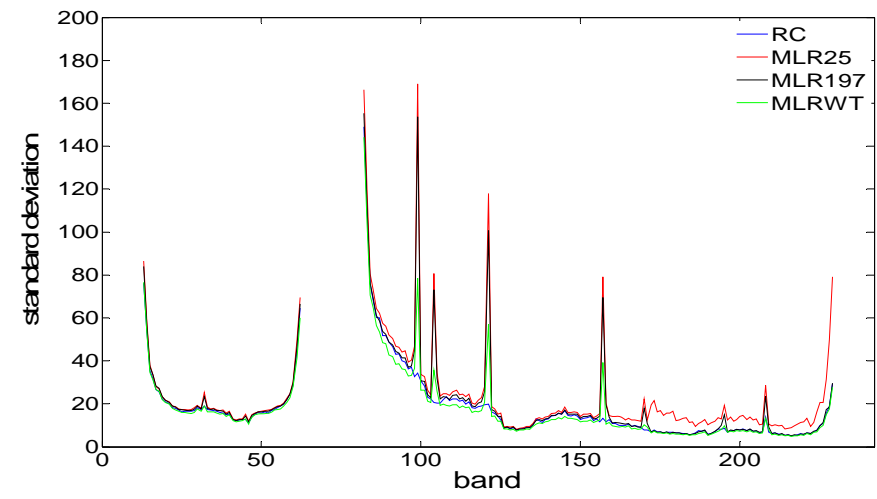

Bol. Ciênc. Geod., sec. Artigos, Curitiba, v. 19, no 4, p.639-652, out-dez, 2013. 
Figure 6 - The spectral correlation coefficient of (a) Jasper Ridge and (b) the Hyperion data. The gaps refer to the bands discarded in the test.

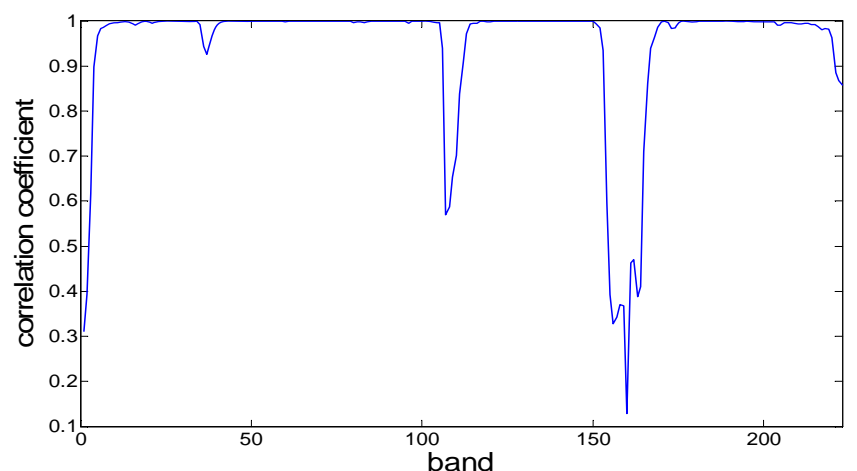

(a)

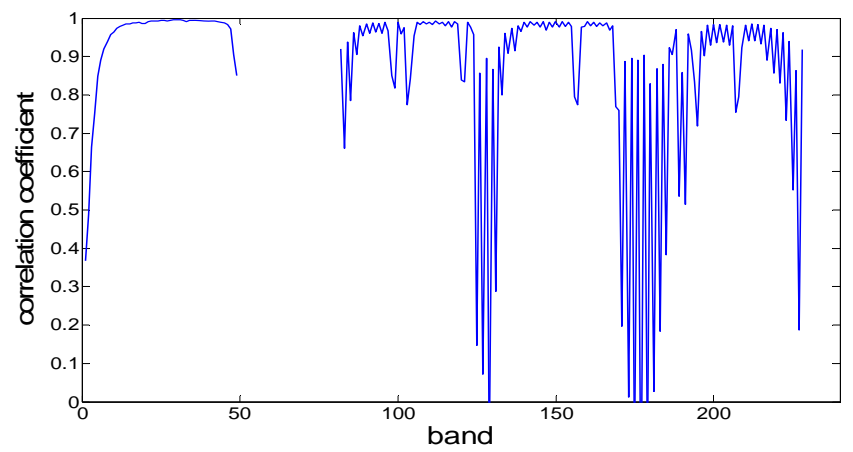

(b)

Figure 5 is the estimated noise standard deviation of different methods for the real data. It shows that the proposed algorithm MLRWT obtains an available estimation result, which indicates that MLRWT method is feasible.

In both simulated experiment and the real data experiment, it is observed that the performance of estimation in some bands is poor, and the estimated standard deviation in some bands changes sharply, even in the adjacent bands, which is not reliable. The reason for this phenomenon is that the spectral correlation in these bands is weak. The spectral correlation coefficient of band $k$ and $k+1$ is defined as

$$
h_{k}=\frac{\sum_{j}^{M} \sum_{i}^{N}\left(x_{i, j}^{k}-u_{k}\right) \times\left(x_{i, j}^{k+1}-u_{k+1}\right)}{\sqrt{\left(\sum_{j}^{M} \sum_{i}^{N}\left(x_{i, j}^{k}-u_{k}\right)^{2}\right) \times\left(\sum_{j}^{M} \sum_{i}^{N}\left(x_{i, j}^{k+1}-u_{k+1}\right)^{2}\right)}}
$$


where $u_{k}$ is the average of pixel values in the $k-t h$ band $k=1,2, \ldots, 223$.

Figure 6 shows the spectral correlation coefficient of the Jasper Ridge and the Hyperion data. Compared with the estimated results in Figure 3, Figure 4 and Figure 5 , it is shown that when the correlation of bands is weak, the performance of estimation based on MLR is poor. However, due to wavelet transform in spatial domain, our method achieves best results among these methods.

\section{CONCLUSION}

The significant spectral correlation of hyperspectral remote sensing image is the theoretical basis of using MLR model. Although the spatial correlation of hyperspectral remote sensing image is weaker than the spectral correlation, it can not be ignored, according to which we use the wavelet transform to remove spatial correlation in order to achieve better noise estimation.

In this paper, we carry out simulated experiments of noise estimation on AVIRIS images and real data experiment on Hyperion data. The MLR removes the spectral correlation while the wavelet transform removes the spatial correlation, which guarantees the performance of noise estimation. As the noise estimation based on classification depends on the content of the image, these methods, such as the SSDC, HRDSDC and RC, the improvement of estimation precision is not significant enough. The RC overcomes the defective segmentation process and prevents the same type of Earth objects that are adjacent in one image into two or more different classes. But it is not able to prevent separating the same type of Earth objects that are not adjacent in one image into two or more different classes. In this paper, MRLWT does not require classification, so it has better adaptability. In the simulated experiment the precision of estimation improves over $39 \%$ in terms of the average error, compared with the general MLR and RC. The new method also overcomes the insensitivity to the spatial characteristic of the classification methods.

In this paper, we only consider the noise term can be properly modelled as additive and spatially stationary. It is reported that the in new-generation hyperspectral sensors the photon noise contribution can not be ignored (Actio, et al, 2011; Uss, et al, 2011), which is not taken account into our method. In future works, we intend to estimate the noise consisting of both signal-dependent and signalindependent terms.

\section{ACKNOWLEDGMENT}

This work is supported by the National Natural Science Foundation of China under Project 61101183 and Project 41201363.

\section{BIBLIOGRAPHICAL REFERENCES}

ACITO, N.; DIANI, M.; CORSINI, G. Signal-Dependent Noise Modeling and Model Parameter Estimation in Hyperspectral Images. IEEE Transactions on Geoscience and Remote Sensing, 49, (8), p. 2957-2971, 2011. 
ACITO, N.; DIANI, M.; CORSINI, G. Residual striping reduction in hyperspectral images. 17th International Conference on Digital Signal Processing (DSP), 2011, p. 1-7, 2011.

BIOUCAS-DIAS, J. M.; NASCIMENTO, J. M. P.; Hyperspectral Subspace Identification. IEEE Transactions on Geoscience and Remote Sensing. 46, (8), p. $2435-2445,2008$.

BRUNO A. et al. Noise modelling and estimation of hypersonic data from airborne imaging spectrometers. Annals of Geophysics, 49, (1), p. 1-9, 2006.

CHEN G. Y.; QIAN S. E. Denoising of Hyperspectral Imagery Using Principal Component Analysis and Wavelet Shrinkage. IEEE Transactions on Geoscience and Remote Sensing. 49, (3), p. 973-980, 2011.

DONOHO, D. Nonlinear wavelet methods for recovery of signals, desities, and apectra from in direct and noisy data. Proceeding of Symposia in Applied Mathematics, p. 173-205, 1993.

DONOHO, D. L.; JOHNSTONE, I. M. Threshold selection for wavelet shrinkage of noisy data. Engineering in Medicine and Biology Society, 1994, p. A24-A25, 1994.

GAO, B. C. An operational method for estimating signal to noise ratios from data acquired with imaging spectrometers. Remote Sensing of Environment, 43, (1), p. 23-33, 1993.

GENG X. R.; ZHANG X.; CHEN Z. G. et al. Classification algorithm based on spatial continuity for hyperspectral image. Journal Infrared Millimeter and Waves, , 23, (4), p. 299-302, 2004

RICHARDS J. A.; JIA X. Remote Sensing Digital Image Analysis, Springer-Verlag, Berlin, 1999, Third Edition.

KESHAVA, N.; MUSTARD, J. F.; Spectral unmixing. IEEE Signal Processing Magazine, 19, (1), p. 44-57, 2002.

KRUSE, F. A.; BOARDMAN, J. W.; HUNTINGTON, J. F. Comparison of airborne hyperspectral data and EO-1 Hyperion for mineral mapping. IEEE Transactions on Geoscience and Remote Sensing, 41, (6), p. 1388-1400, 2003

GAO L. R. et al. A New Operational Method for Estimating Noise in Hyperspectral Images. IEEE Geoscience and Remote Sensing Letters, 5, (1), p. 83-87, 2008.

MALLAT, S. G. A theory for multiresolution signal decomposition: the wavelet representation. IEEE Transactions on Pattern Analysis and Machine Intelligence, 11, (7), p. 674-693, 1989.

JULIO M. J. Comments on "A New Operational Method for Estimating Noise in Hyperspectral Images". IEEE Geoscience and Remote Sensing Letters, 5, (4), p. 705-709, 2008.

MEOLA, J.; EISMANN, M. T.; MOSES, R. L.; ASH, J.N. Modeling and estimation of signal-dependent noise in hyperspectral imagery. Appl. Opt., 50, (21), p. 3829-3846, 2011. 
MIELIKAINEN, J.; TOIVANEN, P. Clustered DPCM for the lossless compression of hyperspectral images. IEEE Transactions on Geoscience and Remote Sensing, 41, (12), p. 2943-2946, 2003.

OTHMAN, H.; QIAN S. E. Noise reduction of hyperspectral imagery using hybrid spatial-spectral derivative-domain wavelet shrinkage. IEEE Transactions on Geoscience and Remote Sensing, 44, (2), p. 397-408, 2006.

ROGER, R. E.; ARNOLD, J. F. Reliably estimating the noise in AVIRIS hyperspectral images. International Journal of Remote Sensing. 17, (10), p. 1951-1962, 1996.

USS, M. L.; VOZEL, B.; LUKIN, V. V.; CHEHDI, K. Local Signal-Dependent Noise Variance Estimation From Hyperspectral Textural Images. IEEE Journal of Selected Topics in Signal Processing, 5, (3), p. 469-486, 2011

(Recebido em abril de 2013. Aceito em julho de 2013). 